\title{
Modalidades de razonamiento en díadas durante la resolución de problemas lógicos
}

\section{Modalities of reasoning in dyads during problem solving} \section{(1) PSicOCGENTE}

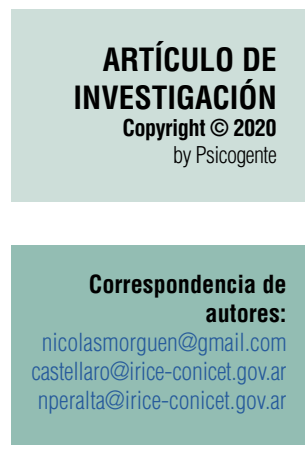

Recibido: $13 / 02 / 19$ Aceptado: $12 / 08 / 19$ Publicado: $01 / 01 / 20$

\author{
Nicolás Morguen (iD - Mariano Castellaro (iD - Nadia Soledad Peralta iD \\ Instituto Rosario de Investigaciones en Ciencias de la Educación (IRICE-CONICET), Rosario, Argentina \\ Universidad Nacional de Rosario (UNR), Rosario, Argentina
}

\section{Resumen}

Objetivos: a) Identificar cualitativamente modalidades de razonamiento en díadas que resuelven problemas lógicos; b) explorar si estas varían en función de tres tipos de composición diádica: dos compañeros de baja competencia (simetría baja), dos compañeros de alta competencia (simetría alta) y un compañero de baja competencia junto a otro de alta competencia (asimetría).

Método: Participaron 36 díadas (72 sujetos, edad: $M=11$ años, 3 meses; $D E=7$ meses), de alumnos de quinto y sexto grado de primaria de Rosario (Argentina) La tarea consistió en una serie de diez ítems lógicos de dificultad creciente (matrices progresivas). Esta fue realizada de dos maneras: primero, de modo individual; segundo, bajo una modalidad colaborativa (en díada). El análisis de contenido se concentró en ítems seleccionados intencionalmente y estuvo basado en la construcción recursiva de categorías (método comparativo constante).

Resultados: El análisis de las interacciones permitió identificar seis modalidades básicas de razonamiento, que fueron desde un nivel inicial basado en una selección arbitraria de la respuesta, hasta un nivel de alta complejidad basado en la construcción de sentido lógico integrando filas y columnas. Por otra parte, las modalidades más rudimentarias (ensayo-error) predominaron en las díadas con simetría baja (40,0\%), mientras que las más avanzadas (de construcción de sentido entre filas y/o columnas) así lo hicieron en las díadas asimétricas $(47,4 \%)$.

Conclusiones: Los resultados permiten ahondar en la comprensión de los procesos sociocognitivos entre pares, especialmente en relación a los fundamentos lógicos propuestos en la actividad. Al mismo tiempo, se discute el concepto de modalidad de razonamiento y se lo diferencia del concepto de modalidad sociocognitiva.

Palabras clave: interacción sociocognitiva; resolución de problemas; infancia; educación primaria; simetría cognitiva; asimetría cognitiva.

\section{Abstract}

Objetives: (a) To identify qualitatively modalities of reasoning in dyads that solve logical problems; b) to explore if they vary depending on three types of dyadic composition: two low-competence partners (low-competence symmetry), two high-competence (high-competence symmetry); a low-competence subject with a high-competence subject (asymmetry).

Method: Participants were 36 dyads ( 72 subjects, age: $M=11$ years old, 3 months; $S D=7$ months) of 5 th and 6 th grade of elementary school, from Rosario (Argentina). The task consisted of a series of ten logical items of increasing difficulty (progressive matrices). This was performed in two ways: first, individually; then, collaborative modality (dyad). The content analysis was concentrated on intentionally selected items and was based on the recursive construction of categories (constant comparative method).

Results: The analysis of interactions allowed identifying six basic modes of reasoning, that were from an initial level based on an arbitrary selection of the answer, up to a level of high complexity based on logical sense construction integrating rows and columns. Moreover, the most rudimentary modalities (trial and error) predominated in the low-competence symmetry dyads (40.0\%), while the most advanced (construction of sense between rows and/or columns) predominated in the asymmetric dyads (47.4\%).

Conclusions: The results allow to depth in the understanding of peer socio-cognitive processes, especially in relation to the logical fundaments proposed in the activity. At the same time, the concept of modality of reasoning is discussed and it is differentiated from the concept of socio-cognitive modality.

Keywords: socio-cognitive interaction; problem solving; childhood; primary school, cognitive symmetry, cognitive asymmetry.

Cómo citar este artículo (APA):

Morguen, N.; Castellaro, M. \& Peralta, N. S. (2020). Modalidades de razonamiento en díadas durante la resolución de problemas lógicos.

Psicogente 23(43), 1-28. https://doi.org/10.17081/psico.23.43.3092 


\section{INTRODUCCIÓN}

Las investigaciones sobre aprendizaje y desarrollo, sostenidas en una perspectiva interaccionista y constructivista, han insistido en la necesidad de considerar los procesos involucrados en la construcción intersubjetiva del conocimiento, más allá de los resultados derivados de esta interacción. Basta con repasar los trabajos pioneros de Perret Clermont, Perret y Bell (1991) o Doise (1986), para notar que estos autores ya habían reparado en dicha cuestión décadas atrás. Hasta ese momento, la mayor parte de los estudios se enfocaba en el efecto cognitivo individual (resultado) de la interacción. A nivel metodológico esto implicaba concentrarse especialmente en comparar el desarrollo de una capacidad o habilidad específica, antes y después de la interacción (Castellaro \& Dominino, 2011).

Los trabajos clásicos mencionados anteriormente se constituyeron en el punto de partida para la segunda y tercera generación de estudios (Psaltis, Duveen \& Perret Clermont, 2009). Estas proponen un cambio fundamental en el foco de análisis: si bien la relación entre interacción social y desarrollo cognitivo (o aprendizaje) constituye aún el sentido teórico básico de las investigaciones, su comprensión cabal implica necesariamente la consideración del proceso de construcción intersubjetiva. De igual manera, la literatura especializada más reciente es heredera de esta idea de base y evidencia un consenso general acerca de la importancia de una mayor profundización y énfasis en la consideración de dicha cuestión.

En este contexto, el objeto de investigación de este artículo ha sido el proceso de razonamiento de díadas en la resolución de problemas lógicos. Esto significa que se trató de estudiar la forma y/o el tipo de fundamento propuesto para la solución de un problema, realizado conjuntamente por dos compañeros y bajo una consigna de trabajo explícitamente colaborativa. En este trabajo, el concepto de intersubjetividad es entendido en sentido amplio, en tanto alude al sistema social, primario e irreductible, que se genera desde el momento en que dos o más sujetos forman parte de una misma actividad, sostenida desde el lenguaje (Castellaro, 2017). Por supuesto, ello no implica desconocer que puede haber formas de interacción más y menos integradas social y cognitivamente (por ejemplo, no es lo mismo que un sujeto aporte todas las ideas y su compañero las reciba pasivamente, a que ambos sujetos aporten diferentes ideas y logren una solución conjunta). Sin embargo, se entiende que aún las situaciones "menos colaborativas" poseen un componente intersubjetivo de base. 
La literatura registra diferentes antecedentes referidos a la interacción entre pares en tareas de razonamiento o cognitivas. Existen investigaciones en tareas inspiradas en la psicología genética piagetiana (Piaget, 1923/2002), asociadas a la construcción de nociones lógicas primarias del desarrollo cognitivo (especialmente, la operacionalidad lógica). Así, hay estudios sobre rotación de planos (Psaltis, 2011; Zapiti \& Psaltis, 2012), balance entre pesos (Denessen, Veenman, Dobbelsteen \& Van Schilt, 2008), trasvasamiento de líquido (Psaltis \& Duveen, 2006, 2007), razonamiento moral (Garton \& Harvey, 2006; Leman y Duveen, 2003), autoconcepto (Campo Ternera, 2014) y desarrollo incipiente de hipótesis (Collantes de Laverde \& Escobar Melo, 2015). En general, estas tareas son utilizadas en un momento evolutivo de pasaje de una lógica pre-operacional hacia una lógica operacional, en el sentido piagetiano del término.

Por otra parte, cuando la interacción entre pares es estudiada en niños de mayor edad, o sea, que ya cuentan con una lógica operatoria claramente instalada (incluso con características formales, como ocurre habitualmente hacia finales de la educación primaria), una tarea muy utilizada en la literatura fueron las matrices progresivas (Castellaro \& Roselli, 2018a, 2018b; Fernández, Wegeriff, Mercer \& Rojas-Drummond, 2001; Rojas-Drummond, Mazón, Fernández \& Wegerif, 2006; Rojas-Drummond \& Mercer, 2003; Rojas-Drummond \& Peón Zapata, 2004; Roselli, 2004, 2010; Roselli, Bruno \& Evangelista, 2004a, 2004b; Wegerif et al., 2016; Webb \& Treagust, 2006; Yang, 2015). Lo anterior fundamentó la utilización de dicha tarea en el presente trabajo. Estos antecedentes utilizan ese formato de actividad como base para el análisis de la interacción sociocognitiva, en tanto constituye un dispositivo que activa y permite el estudio de procesos intersubjetivos de construcción de soluciones lógicas. En ese sentido, en lo que hace propiamente a este tipo de estudios, el uso de esta actividad se aleja de su propósito fundacional que fue establecer psicométricamente el nivel de inteligencia general individual (por ejemplo, Raven, 1991).

En general, la tarea está compuesta por varios ítems, cada uno de los cuales implica un problema particular. Un ítem consiste en un conjunto de figuras, ordenadas en filas y columnas, e interconectadas por una relación de sentido. Al mismo tiempo, la última figura que completaría la secuencia lógica está ausente. Precisamente, la resolución del ítem implica deducir la relación de sentido lógico común a las figuras y, consiguientemente, la figura ausente que completa dicha secuencia. Para ello, se dispone de una serie de opciones de respuestas entre las cuales se encuentra la correcta. 
Algunos trabajos utilizaron una adaptación especial del Test de Matrices Progresivas de Raven (1991), que consistió en dividir la versión original de 60 ítems en dos versiones paralelas de 30. Tal fue el caso de Fernández et al. (2001), Rojas-Drummond et al. (2006), Rojas-Drummond y Mercer (2003), Rojas-Drummond y Peón Zapata (2004), Wegerif et al. (2016), Webb y Treagust (2006) y Yang (2015). Así, disponían de versiones paralelas para aplicarla en diferentes condiciones (por ejemplo, resolución individual y en colaboración), logrando un control metodológico de las características de la tarea. Por otro lado, un segundo grupo de investigaciones (Castellaro \& Roselli, 2018a, 2018b; Roselli, 2004, 2010; Roselli et al., 2004a, 2004b) desarrolló otras adaptaciones de la tarea original, utilizando menor cantidad de ítems y, en algunos casos, construyendo ítems ad hoc, además de utilizar versiones paralelas de la tarea.

Más allá de las diferencias entre ambas posiciones metodológicas previas, todos los estudios cuentan con un diseño similar. En una fase inicial, los sujetos realizan individualmente la tarea, para contar con un indicador de su grado de competencia específica en ese tipo de actividad. En función de dicha evaluación inicial, se conforman díadas cuyos integrantes cuentan con similares o diferentes niveles de competencia individual, las cuales realizan la misma actividad (o una versión equivalente) de manera colectiva. En esta fase se concentran los principales análisis, relativos a la interacción sociocognitiva. Una última etapa, presente solo en antecedentes puntuales (no ocurrió en el presente artículo), consiste en repetir la realización individual de la tarea para estudiar el progreso cognitivo como producto de la interacción, basando los estudios en un enfoque resultadista (Brizuela \& Scheuer, 2016).

El énfasis de todos estos trabajos estuvo puesto en analizar modalidades interactivas o sociocognitivas, es decir, formas en que los compañeros coordinan sus acciones para arribar al objetivo planteado por la tarea. En este sentido, uno de los modelos teóricos más utilizados fue el propuesto por Mercer, basado en la diferenciación entre tres tipos de interacción verbal: exploratorio, de disputa y acumulativo (Fernández et al., 2001; RojasDrummond et al., 2006; Rojas-Drummond \& Mercer, 2003; Rojas-Drummond \& Peón Zapata, 2004; Schmitz \& Winskel, 2008; Wegerif et al., 2016; Webb \& Treagust, 2006; Yang, 2015). El primero (exploratorio) está caracterizado por la auténtica construcción de significados compartidos a partir del ofrecimiento de sugerencias y alternativas de acción, sumado a la confrontación y puesta a prueba de las ideas. Esto se diferencia claramente de las otras dos formas de interacción. Por un lado, el patrón de disputa implica el predo- 
minio de desacuerdo constante y la toma individual de decisiones, sumado a la dificultad para comprender el punto de vista ajeno. Por el otro, en el patrón acumulativo, los compañeros logran acuerdos básicos sobre la resolución de la tarea y cierto grado de construcción compartida del conocimiento; sin embargo, esto se da por una adhesión acrítica respecto a lo dicho por el compañero (solo se repite o confirma sin reflexión).

Tal como ya se mencionó, la mayor parte de estos antecedentes se enfocó en las características de la modalidad sociocognitiva. Ahora bien, el eje del presente trabajo, si bien guarda relación con ello, estuvo más bien en la forma de razonamiento propuesto para resolver un problema, o sea, el fundamento propuesto por la díada para proponer una solución al problema, lo cual no cuenta con desarrollos previos. En otras palabras, el artículo no refiere al estudio de modalidades de razonamiento colaborativo, sino que simplemente apunta a detectar diferentes formas de fundamentación de la solución en un contexto diádico. Esto, sumado a la intención de efectuar un microanálisis de dicho proceso, propició que la investigación adoptara un enfoque metodológico global de carácter cualitativo-inductivo, es decir, que apunte a la identificación ad hoc de diferentes modalidades de razonamiento diádico. En otras palabras, a partir del análisis procesual se intentó construir un conjunto de categorías de sentido relativas a modalidades de razonamiento colectivo (Glasser \& Strauss, 1967/2006; Strauss \& Corbin, 1998).

Al propósito principal ya mencionado, también se agregó un análisis secundario relativo a la composición sociocognitiva de la díada. Este último término alude a la simetría o asimetría (igualdad o diferencia) entre las competencias específicas individuales de los compañeros de díada (Castellaro \& Roselli, 2018a, 2018b). El estudio de esta variable cuenta con múltiples antecedentes. Esto se explica por el hecho de que, generalmente, la interacción colaborativa es estudiada en el marco de una tarea puntual (por su relación con una capacidad o habilidad determinada) y, por tanto, es postulable que el grado de igualdad o desigualdad entre las competencias específicas de los compañeros determine una particular modalidad interactiva. En consecuencia, si bien el foco inicial del trabajo estuvo en identificar cualitativamente modalidades de razonamiento diádico, también interesó explorar si la presencia de dichas modalidades de razonamiento variaba en función de tres tipos de composición diádica: dos compañeros de baja competencia (simetría baja), dos compañeros de alta competencia (simetría alta) y un compañero de baja competencia junto a otro de alta competencia (asimetría). 
En relación a la cuestión de la simetría y asimetría cognitiva, es posible identificar dos posiciones teóricas. La perspectiva neopiagetiana (Psaltis \& Duveen, 2006, 2007; Zapiti \& Psaltis, 2012) privilegia el intercambio entre individuos con similares niveles de competencia inicial, aunque con diferentes perspectivas sobre un mismo problema, condición básica inicial para el conflicto sociocognitivo (diferencias cualitativas). Por su parte, la perspectiva neovygostkiana (Asterhan, Schwarz \& Cohen-Eliyahu, 2014; Fawcett \& Garton, 2005; Gabriele, 2007; Garton \& Pratt, 2001; Schmitz \& Winskel, 2008) destaca sobremanera el valor de la diferencia de grado entre competencias, siempre y cuando esta sea moderada y no extrema.

El presente trabajo propuso dos objetivos. El primero, de carácter principal, fue identificar modalidades de razonamiento en díadas que resuelven problemas lógicos. A esto se agregó un objetivo secundario, explorar si la presencia de modalidades de razonamiento (coincidentes con segmentos de interacción; esto se definirá en la descripción de la metodología) varían en función de tres tipos de composición diádica: dos compañeros de baja competencia (simetría baja), dos compañeros de alta competencia (simetría alta) y un compañero de baja competencia junto a otro de alta competencia (asimetría).

El carácter básicamente cualitativo-inductivo del estudio se fundamentó en la necesidad de estudiar a la construcción sociocognitiva de la manera más dinámica, clínica y procesual posible. Por ese motivo, el eje principal fue la identificación ad hoc de diferentes modalidades de razonamiento diádico, a partir de la inmersión y movimiento recursivo sobre el conjunto de los datos (Glasser \& Strauss, 1967/2006; Strauss \& Corbin, 1998), lo cual es poco frecuente dentro de la literatura especializada. Aun así, el trabajo estuvo guiado por dos presupuestos globales (que no constituyeron hipótesis en el sentido clásico del término): a) dentro de un proceso colaborativo entre pares es posible reconocer diferentes formas (incluso, niveles) de razonamiento en la co-construcción de la solución del problema; b) la igualdad o diferencia entre los niveles de competencia específica de los compañeros de díada se asocia con la presencia de modalidades de razonamiento específicas.

\section{MÉTODO}

\subsection{Participantes}

Participaron 36 díadas ( 72 sujetos; edad en años, meses: $M=11,3, S D=0,3$ ). El $69,4 \%$ de la muestra estuvo conformado por niñas. Todas las díadas estaban 
integradas por dos compañeros del mismo género. Todos eran alumnos de quinto y sexto grado de dos escuelas de la zona céntrica de la ciudad de Rosario (Argentina), ambas de gestión pública y con un alumnado de nivel socioeconómico medio en general. De la muestra inicialmente disponible, se excluyeron los casos sin consentimiento informado del responsable adulto o del propio niño, al igual que los casos atípicos. Esto último aludió a estudiantes repitentes, con dificultades marcadas de aprendizaje y/o con diagnóstico profesional de retraso en algún área del desarrollo psicológico.

\subsection{Materiales y procedimiento}

El proyecto contó con la evaluación y autorización ética correspondiente. Se obtuvieron los consentimientos informados de las autoridades de cada escuela, los padres de los alumnos y los propios sujetos. No participaron de la experiencia aquellos alumnos que no contaron con alguno de estos permisos. La tarea consistió en una serie de diez ítems lógicos de dificultad creciente, extraídos y/o adaptados de la Escala General del Test de Matrices Progresivas (Raven, 1991). Dentro de la literatura sobre interacción sociocognitiva entre pares, el uso de esta tarea apunta a construir un dispositivo o situación activadora de procesos lógicos, que son estudiados en un marco interactivo (esto implica un alejamiento con respecto a su función psicométrica original, de medición de la inteligencia). Así, se propuso la cantidad específica de diez ítems con el propósito de contar con una apreciación general de dichos procesos sociocognitivos a lo largo de un set de problemas, evitando -al mismo tiempo- el desgaste motivacional o cansancio excesivo de los participantes. Esta misma cantidad de ítems también se utilizó para la fase individual inicial, con el objetivo de mantener constante las condiciones de observación del fenómeno, tanto en la etapa individual como en la etapa posterior colectiva (Castellaro \& Roselli, 2018a, 2018b; Roselli, 2004, 2010; Roselli et al., 2004a, 2004b).

Cada ítem estaba constituido por ocho figuras (en realidad, la novena estaba ausente) distribuidas en tres filas y tres columnas, que mantenían una relación de sentido lógico entre sí. Así, la resolución de cada ítem implicaba deducir esta relación de sentido entre las ocho figuras presentes y detectar la novena figura ausente. Para ello, se disponía de una serie de ocho opciones de respuesta, entre las cuales figuraba aquella que completaba de manera correcta la secuencia lógica. La correcta y auténtica resolución de cada ítem implica deducir relaciones lógicas en dos sentidos posibles: por un lado, únicamente por filas o únicamente por columnas (uno de ambos); por el otro, 
logrando la integración de las lógicas por filas y por columnas, lo cual supone un nivel más complejo y global de elaboración cognitiva que el anterior.

La tarea fue realizada bajo dos modalidades diferentes. En una primera instancia, fue resuelta de modo individual por cada sujeto; en este caso, también se agregaron dos ítems adicionales de ensayo inicial. Esta fase se realizó en el aula de clase de cada curso, en el horario de cursado habitual (en turno mañana o tarde, según el caso), y llevó un tiempo aproximado de entre 15 y 25 minutos. En las dos o tres semanas posteriores, una versión paralela de la tarea (ítems equivalentes) fue realizada por cada díada (fase interactiva). En esta etapa, una o algunas díadas (tres como máximo) eran convocadas simultáneamente para realizar la actividad. Esto se dio en un aula disponible (por ejemplo, disponible para usos múltiples o informática), diferente al aula de cursado habitual, lo cual también ocurrió durante el horario de clase. La resolución diádica duraba entre 15 y 30 minutos, según el caso. Durante el estudio completo, dos o tres investigadores estuvieron presentes, a los fines de explicar las consignas de trabajo, observar la actividad y asegurar el registro de audio de la interacción (en el caso de la fase interactiva).

La equivalencia de ambas versiones de la serie fue determinada a partir de una prueba piloto anterior al estudio propiamente dicho, con sujetos de cursos similares. Estos resolvieron dos series con mayor número de ítems a los aplicados en este trabajo (con contrabalanceo del orden de administración). A partir de esta medición, se constituyeron dos versiones definitivas integradas por diez pares de ítems que no habían registrado diferencias significativas entre sí en cuanto al grado de acierto/error (acierto=1, error=0; prueba T de Student para muestras relacionadas, $p=n . s$.$) .$

La evaluación individual inicial se utilizó como criterio para la conformación posterior de las díadas respectivas (simétricas de baja y alta competencia, más las asimétricas). El nivel de competencia individual bajo o alto se determinó a partir de la distribución de frecuencias del total de ítems correctos de cada sujeto: nivel bajo ( 0 a 5 puntos) y nivel alto ( 6 a 10 puntos). Como criterio complementario, las díadas simétricas estuvieron integradas por sujetos con una diferencia de dos puntos o menos entre sí, mientras que las asimétricas por una diferencia de cuatro o más puntos. Así, se constituyeron tres tipos de díada: simétricas de baja competencia $(n=10)$, simétricas de alta competencia $(n=15), y$ asimétricas $(n=11)$. La consigna colaborativa sugería a los compañeros de díada dialogar e intercambiar ideas sobre posibles soluciones del ítem y consensuar una decisión final al respecto. La interacción fue regis- 
trada vía audio y transcrita. La codificación del material se realizó sobre las grabaciones obtenidas.

\subsection{Análisis de los datos en ítems específicos}

El trabajo propuso un análisis minucioso de la modalidad de razonamiento diádica durante la resolución de ítems lógicos. Esto implicó un abordaje microanalítico, basado en la selección intencional de ítems prototípicos sobre los cuales se aplicaron los análisis correspondientes. Este proceso de selección de ítems prototípicos tuvo dos etapas sucesivas (doble filtro), que se describen a continuación.

Primero, en las díadas con simetría general (es decir, donde ambos sujetos habían registrado puntajes totales individuales de dos o menos puntos de diferencia entre sí) se consideraron inicialmente los ítems donde cada sujeto había mostrado una respuesta errónea individual. En cambio, en las díadas con asimetría general (es decir, donde ambos sujetos habían registrado puntajes totales individuales de cuatro o más puntos de diferencia entre sí) se consideraron inicialmente los ítems específicos donde el sujeto de mayor competencia general había mostrado una respuesta correcta individual y el sujeto de menor competencia general había mostrado una respuesta incorrecta individual. De esta forma, se intentó seleccionar ítems que reproducían a nivel específico (micro) la igualdad o desigualdad cognitiva general captada al inicio del estudio.

De los ítems escogidos en el paso anterior, se observó que algunos eran aplicables a la mayoría de las díadas estudiadas, mientras que otros eran aplicables en muchos menos casos (presencia más atípica dentro de la muestra). El segundo paso selectivo consistió en tomar solamente los ítems del primer grupo, los cuales podían ser el 6, el 8, el 9 y el 10 de la serie original (ver Anexo). Dichos ítems representaban los de mayor dificultad dentro de la serie completa, lo cual se asoció con las interacciones más ricas, ya sea por la extensión y variedad de mensajes, como por la variedad de propuestas de solución. Esto constituyó un elemento intencional complementario que justificó la elección de los ítems analizados.

Por lo tanto, en cada una de las 36 díadas se observó si el ítem 6, 8, 9 o 10 cumplía con los criterios básicos para incluirse en el análisis definitivo. Así, quedaron seleccionados 90 ítems de los 360 inicialmente disponibles (considerando que cada una de las 36 díadas había realizado la serie de 10 ítems), los cuales constituyeron el corpus definitivo analizado. Del total de ítems 
seleccionados, 35 correspondieron a díadas con simetría baja, 27 a díadas con simetría alta y 28 a díadas asimétricas.

Al interior de cada ítem seleccionado, se analizó o analizaron la/s modalidad/ es de razonamiento propuesta/s por la díada durante la resolución de ese problema en particular. En un mismo ítem podía registrarse más de una modalidad conversacional de razonamiento, en tanto cada una de estas se aplicaba a un segmento de interacción. Este consistía en un fragmento de intercambio verbal (categoría molar), cuyo comienzo estaba determinado por una enunciación que daba cuenta de una lógica o modalidad particular de razonamiento; su finalización se decidía cuando comenzaba una lógica nueva o simplemente por la propia resolución final del ítem. Ello implicó que los segmentos tuvieran extensiones variables entre sí.

La identificación y categorización de las diferentes modalidades de razonamiento se basó en los principios generales del procesamiento cualitativo y el método de comparación constante (Glasser \& Strauss, 1967/2006; Strauss \& Corbin, 1998). A partir de reiteradas lecturas del material se comenzaron a definir categorías globales, que se fueron especificando, diferenciando y/o ajustando entre sí a medida que se avanzaba en la relectura del material. Finalmente, el cierre del proceso de categorización se determinó atendiendo al principio de saturación teórica, el cual indica el detenimiento del análisis cuando los nuevos datos ya no aportan información adicional sobre las categorías construidas (Cornejo \& Salas, 2011; Soler Pujals \& Enrique Jiménez, 2012; Twining, Heller, Nussbaum \& Chung Tsai, 2017).

\subsection{Conflicto de interés}

Los autores declaran que el trabajo no está asociado a conflicto de interés alguno.

\section{RESULTADOS}

\subsection{Modalidades de razonamiento identificadas durante la resolución diádica de los ítems}

El análisis de las interacciones permitió identificar seis modalidades básicas de razonamiento, que fueron desde un nivel inicial basado en una selección arbitraria de la respuesta hasta un nivel de alta complejidad basado en la construcción de sentido lógico integrando filas y columnas. Como se dijo, al interior de la resolución de un ítem en particular, la díada podía atravesar por distintas formas de construcción de la respuesta, las cuales podían tener 
extensiones variables entre sí. A continuación, se presenta cada una de las modalidades identificadas y se describen/analizan fragmentos interactivos casuísticos ilustrativos.

\subsubsection{Selección arbitraria de una respuesta}

Se propone una solución de respuesta de modo directo, es decir, sin ninguna fundamentación o justificación verbal explícita que la avale (tipo ensayo y error). No es posible determinar si el/los sujeto/s cuentan con una justificación interna no explicitada de la solución, pero lo cierto es que esta no se manifiesta a nivel de la interacción registrada.

\subsubsection{Descarte de las opciones de respuesta disponibles}

El fundamento de la respuesta está basado en la detección de opciones de respuesta ya repetidas dentro de las figuras que componen el problema (la secuencia lógica presentada), lo cual hace que las primeras sean descartadas como una solución válida. También puede darse el proceso inverso: que se proponga una opción de respuesta por no estar presente entre las figuras que integran la secuencia lógica. Esto último se ilustra en el siguiente fragmento (en ítem 6).

Tabla 1.

Selección por descarte de las opciones de respuesta disponibles

\begin{tabular}{ccc}
\hline TURNO & SUJETO & MENSAJE \\
\hline 1 & A & El 6 (opción de respuesta)... (observa)... sí está (figura F)... \\
2 & A & El 3 (opción de respuesta)... no está... (entre las figuras del problema) \\
3 & B & No está... (observan)... \\
4 & A & El 7 (opción de respuesta).... no está... \\
5 & B & No está... \\
6 & A & El 4 (opción de respuesta)... (silencio)... no está tampoco.... \\
7 & B & Y el 8 (opción de respuesta)... (observa)...si está (figura H del problema) \\
8 & A & Para mí puede ser el 7 (opción de respuesta)... (silencio) \\
9 & B & No la entiendo, pero no importa... \\
10 & A & Pongamos 7 (anotan) \\
\hline
\end{tabular}

En la ejemplificación se observa cómo se aplica esta lógica en el mensaje 1, donde el sujeto A observa la opción 6 y la descarta por ya estar presente en una de las figuras de la parte superior. En los mensajes 2, 4 y 6 se evalúan las opciones de respuesta 3,7 y 4 bajo la misma lógica; al no percibir estas 
opciones en la parte superior de la hoja se las mantienen como posibles respuestas. Luego, siguiendo la misma idea se descarta la opción 8 por ya estar presente en la figura $\mathrm{H}$. Finalmente, se termina decidiendo la respuesta final (opción 7) por un criterio poco fundamentado, sin apelar en ningún momento a la relación de sentido lógico subyacente en el problema.

\subsubsection{Combinación entre selección arbitraria y por descarte de opciones} de respuesta

Tal como se puede insinuar, esta categoría resulta de la combinación de los dos primeros tipos identificados previamente. Ante la selección arbitraria de la respuesta por parte de uno de los sujetos, el otro responde descartando esa opción porque ya se encuentra presente entre las figuras que conforman la secuencia lógica.

\section{Tabla 2.}

Combinación entre selección arbitraria y por descarte de las opciones de respuesta disponibles

\begin{tabular}{cc}
\hline SUJETO & MENSAJE \\
\hline A & Creo que era el 8. (opción de respuesta; Respuesta arbitraria) \\
B & El 8 no es, porque el 8 es el E. (Figura del problema. Descarte). \\
\hline
\end{tabular}

El fragmento (en ítem 8) muestra que en un primer momento uno de los sujetos selecciona una posible opción de manera arbitraria (donde solo se expresa la respuesta elegida sin dar ningún fundamento que justifique dicha elección, como fue señalado en la definición). Luego, la lógica que se emplea para responder deriva del segundo nivel, el mismo supone descartar la opción elegida porque ya se encuentra presente entre las figuras que corresponden al resto de la secuencia. En el ejemplo esto se manifiesta claramente por el sujeto A (que selecciona la respuesta 8 sin comunicar nada más), y por el sujeto $B$ que le responde que eso es imposible por ya encontrarse dicha opción en la zona superior del cuadro, donde se la identifica como la figura E.

\subsubsection{Descripción de figuras del ítem, sin proponer una relación de sentido entre estas}

Las verbalizaciones de la díada refieren a las características (forma, tamaño, etc.) de alguna/s figura/s en particular, pero sin llegar a establecer una relación de sentido lógico entre estas que trascienda dichos aspectos fenomenológicos. El siguiente ejemplo ilustra lo anterior (en ítem 8). 
Tabla 3.

Descripción de figuras del ítem, sin proponer una relación de sentido

\begin{tabular}{|c|c|c|}
\hline TURNO & SUJETO & MENSAJE \\
\hline 1 & A & Acá hay dos triángulos, faltaría un triángulo. \\
\hline 2 & B & $\begin{array}{l}\text { El B es un triángulo...Mira, fijate que el B es un triángulo entero, el F es un triángulo } \\
\qquad \text { sin el palito, } y \ldots\end{array}$ \\
\hline 3 & A & $\begin{array}{l}\text { (Interrumpiendo) entonces hay que buscar un triángulo que no tiene... (se detie- } \\
\text { ne)... no hay }\end{array}$ \\
\hline 4 & B & Pero no hay... \\
\hline 5 & A & ¿Este? (señala opción 7) \\
\hline 6 & B & No, eso no es un triángulo \\
\hline 7 & A & Claro... (ríe) \\
\hline 8 & B & Porque ahí sí, tenés razón...por ahí es el 7 (opción de respuesta) \\
\hline 9 & A & Porque por ahí le sacas esto... (parte superior del triángulo) \\
\hline 10 & B & Claro \\
\hline 11 & A & Pero esto tiene el palito... \\
\hline 12 & B & Claro... \\
\hline 13 & A & Pero no hay ningún otro que se le parezca \\
\hline 14 & B & $\begin{array}{l}\text { Pone el } 7 \text { y listo. (Al final se toma una decisión sin apelar a otro criterio más que a } \\
\text { las descripciones previas) }\end{array}$ \\
\hline
\end{tabular}

Esta modalidad se basa principalmente en una descripción de lo que se visualiza pero sin nombrar una interrelación de sentido entre las figuras. Esta descripción puede ser de todas o de alguna de las figuras, y más o menos detallada, según el grado de rigurosidad de la descripción. Lo que nos permite categorizar a este ejemplo en la modalidad correspondiente es que no se hace referencia a los otros tipos de figuras y a su ubicación, ya que las mismas se presentan una vez por columna y por fila en sus diferentes condiciones (completa, abierta en la parte inferior y abierta en la parte superior), lo cual implicaría una lógica más avanzada tal como las que se describen a continuación.

\subsubsection{Construcción parcial de sentido (por filas o por columnas)}

Modalidad de razonamiento basada en la construcción de un sentido lógico por filas o por columnas, es decir, no hay integración entre ambas. Esta construcción de sentido implica una relación que trasciende los elementos puramente perceptuales de las figuras presentes en el problema. 
Tabla 4.

Construcción parcial de sentido (por filas o por columnas)

\begin{tabular}{|c|c|c|}
\hline TURNO & SUJETO & MENSAJE \\
\hline 1 & A & Fijémonos el diseño que falta.... \\
\hline 2 & B & Si... (observan) \\
\hline 3 & B & $\begin{array}{c}\text { Anda contando... mirá... 2, 3, 4...6,5,4...entendes? (analiza la cantidad de círculos } \\
\text { internos que tiene cada una de las tres figuras de una fila y luego de otra) }\end{array}$ \\
\hline 4 & B & Las filas van formando...mirá... (generalización de la lógica a las tres filas) \\
\hline 5 & A & Si, si, si.... \\
\hline 6 & B & Entonces sería el $5 \ldots$ \\
\hline 7 & A & Si. \\
\hline
\end{tabular}

En el fragmento previo (en ítem 6, constituido por figuras con diferentes cantidades de círculos internos) se observa en el mensaje 3 cómo se visualiza una lógica de filas: "2, 3, 4" refiere a la cantidad de círculos en cada una de las imágenes de la fila superior, siendo estas contadas de derecha a izquierda. Luego se continúa con el enunciado " 6 , 5, 4" que hace referencia a las circunferencias de la fila inferior, es decir que hay 6 circunferencias en la figura G, 5 en la $\mathrm{H}, \mathrm{y}$ que por lo tanto la figura de la respuesta correcta debe tener 4 . Pero pese a lo correcto de esta afirmación, al no realizarse un entrecruzamiento de este dato con la variación de la disposición que se da al ir cambiando de filas y columnas conjuntamente, les resulta dificultoso observar la disposición que debería tener la respuesta correcta. Cabe destacar que lo mismo hubiera pasado si se observaran solo las columnas, ya que lo que brinda el dato de la disposición final de la figura solo se logra al apreciar las relaciones de filas y columnas en su conjunto.

\subsubsection{Construcción global de sentido (por integración entre filas y columnas)}

Modalidad de razonamiento basada en la construcción de un sentido lógico integrando las lógicas de las filas y de las columnas. Esta construcción de sentido implica una relación que trasciende los elementos puramente perceptuales de las figuras presentes en el problema. Representa la modalidad de razonamiento más avanzada registrada en este trabajo. Se presenta un fragmento correspondiente al ítem 6. 
Tabla 5.

Construcción global de sentido (por filas y por columnas)

\begin{tabular}{|c|c|c|}
\hline TURNO & SUJETO & MENSAJE \\
\hline 1 & K & Bueno, en un cuadradito, que sería el A, hay 4 puntos, en el B hay 3, en el $C$ hay 2. En el D hay... \\
\hline 2 & $S$ & (intenta interrumpir) pero fijate... que el A se le saca cómo un circulito, se le saca este, ¿entendés? \\
\hline 3 & K & Sí. \\
\hline 4 & S & Si yo lo tapo (al círculo), A y B son iguales. \\
\hline 5 & K & $\mathrm{Si}$ \\
\hline 6 & S & ¿Viste? \\
\hline 7 & S & Y el C, si yo tapo estos dos (círculos), el A y el C son iguales \\
\hline 8 & K & Sí. \\
\hline 9 & S & ¿Entendés? Entonces cómo se les van sacando. \\
\hline 10 & K & Ajá. \\
\hline 11 & S & Y lo mismo pasa en el D, E y F. O sea, le tapas esto y... \\
\hline 12 & K & ...quedan estos dos iguales. \\
\hline 13 & S & Y quedan estos dos iguales. Le tapas esto y quedan estos dos iguales. \\
\hline 14 & K & Ajá. Y le tendrías que tapar a estos así (la última fila). \\
\hline 15 & S & Sí. \\
\hline 16 & S & $\begin{array}{l}\text { Y entonces, al G si le sacas } 1 \text { queda como el H. Y al H....fijate, primero se le saca el que está en el } \\
\text { vértice de arriba derecho... primero se le saca siempre este, ¿ives? }\end{array}$ \\
\hline 17 & K & Si.... \\
\hline 18 & K & Ah, ya sé cual es... \\
\hline 19 & $S$ & A ver, ¿̇cuál es? (no responde K) \\
\hline 20 & S & El $1 \ldots$ \\
\hline 21 & K & Es verdad, es el 1. \\
\hline 22 & S & Para. Sino, ¿¿cuál otro podría ser? \\
\hline 23 & K & A ver, este tiene dos posibilidades: puede ser el 8 o el 5, como dijiste vos. \\
\hline 24 & S & $\begin{array}{c}\text { Pará, pará, pará... En el del medio siempre se le saca... hay que sacarle...si, si, es el 1, porque en el } \\
\text { tercero, en la línea de la derecha, siempre se le saca el de arriba del medio...¿ives? }\end{array}$ \\
\hline 25 & K & Ajá... \\
\hline 26 & S & Y en este siempre se le saca el del vértice a la derecha de arriba (G) \\
\hline 27 & K & Ajá. \\
\hline 28 & S & Entonces en este se le sacaría el de acá del medio y quedaría como el $1(\mathrm{H})$ \\
\hline 29 & K & Ajá \\
\hline 30 & S & Entonces es el 1 (anotan) \\
\hline
\end{tabular}

En el ejemplo se aprecia la construcción de sentido en el orden de las filas $(A, B, C / D, E, F / G, H)$, consistente en la progresiva eliminación del círculo superior derecho de cada figura. Este hallazgo les permite entender que la respuesta correcta es la opción 1 . Sin embargo, al evaluar otras posibles opciones, en el mensaje 24 se da un suceso clave, ya que es allí donde se agrega la construcción de sentido por columnas (la derecha). Este doble cruce de filas y columnas permite reconfirmar que la respuesta correcta es la número 1. 


\subsection{Modalidades de razonamiento y composición sociocognitiva de la díada}

Al interior de un ítem podía observarse más de una modalidad de razonamiento, lo cual ocurrió en varias díadas. Esto fue así porque cada modalidad de razonamiento coincidía con un segmento de razonamiento, lo cual consistía en un fragmento de intercambio verbal (categoría molar) cuyo comienzo estaba determinado por una enunciación que daba cuenta de una lógica o modalidad particular de razonamiento; su finalización se decidía cuando comenzaba una lógica nueva o simplemente por la propia resolución final del ítem. En total, dentro de los 90 ítems analizados, se registraron 187 segmentos de razonamiento (es decir, al interior de algunos ítems solo se observó un segmento de razonamiento, mientras en otros se observaron más de uno). Cada segmento fue analizado en función de la composición sociocognitiva de la díada (simetría baja, simetría alta o asimetría) en la cual fue observado. En la tabla 6 se presentan los recuentos de tipos de segmentos, en función de la composición sociocognitiva de la díada en la cual fueron hallados.

Tabla 6.

Relación entre modalidades de razonamiento colaborativo (segmentos) y composición de la díada (frecuencias, \% por filas, \% por columnas).

\begin{tabular}{|c|c|c|c|c|c|}
\hline & & SIMETRÍA BAJA & SIMETRÍA ALTA & ASIMETRÍA & TOTAL \\
\hline \multirow{4}{*}{ Selección arbitraria de respuesta } & Recuento & 24 & 20 & 19 & 63 \\
\hline & $\%$ fila & $38,1 \%$ & $31,7 \%$ & $30,2 \%$ & $100,0 \%$ \\
\hline & $\%$ columna & $34,3 \%$ & $32,8 \%$ & $33,9 \%$ & $33,7 \%$ \\
\hline & Recuento & 9 & 6 & 3 & 18 \\
\hline \multirow[t]{2}{*}{ Descarte de las opciones de respuesta } & $\%$ fila & $50,0 \%$ & $33,3 \%$ & $16,7 \%$ & $100,0 \%$ \\
\hline & $\%$ columna & $12,9 \%$ & $9,8 \%$ & $5,4 \%$ & $9,6 \%$ \\
\hline \multirow{4}{*}{$\begin{array}{l}\text { Combinación de selección azarosa y } \\
\text { descarte de opciones de respuesta }\end{array}$} & Recuento & 5 & 6 & 3 & 14 \\
\hline & $\%$ fila & $35,7 \%$ & $42,9 \%$ & $21,4 \%$ & $100,0 \%$ \\
\hline & $\%$ columna & $7,1 \%$ & $9,8 \%$ & $5,4 \%$ & $7,5 \%$ \\
\hline & Recuento & 20 & 21 & 13 & 54 \\
\hline \multirow[t]{2}{*}{ Descripción de figuras } & $\%$ fila & $37,0 \%$ & $38,9 \%$ & $24,1 \%$ & $100,0 \%$ \\
\hline & $\%$ columna & $28,6 \%$ & $34,4 \%$ & $23,2 \%$ & $28,9 \%$ \\
\hline \multirow{3}{*}{$\begin{array}{l}\text { Construcción de sentido parcial (fila o } \\
\text { columna) }\end{array}$} & Recuento & 6 & 4 & 7 & 17 \\
\hline & $\%$ fila & $35,3 \%$ & $23,5 \%$ & $41,2 \%$ & $100,0 \%$ \\
\hline & $\%$ columna & $8,6 \%$ & $6,6 \%$ & $12,5 \%$ & $9,1 \%$ \\
\hline \multirow{4}{*}{$\begin{array}{l}\text { Construcción de sentido global (fila + } \\
\text { columna) }\end{array}$} & Recuento & 6 & 4 & 11 & 21 \\
\hline & $\%$ fila & $28,6 \%$ & $19,0 \%$ & $52,4 \%$ & $100,0 \%$ \\
\hline & $\%$ columna & $8,6 \%$ & $6,6 \%$ & $19,6 \%$ & $11,2 \%$ \\
\hline & Recuento & 70 & 61 & 56 & 187 \\
\hline \multirow[t]{2}{*}{ TOTAL } & $\%$ fila & $37,4 \%$ & $32,6 \%$ & $29,9 \%$ & $100,0 \%$ \\
\hline & $\%$ columna & $100,0 \%$ & $100,0 \%$ & $100,0 \%$ & $100,0 \%$ \\
\hline
\end{tabular}


Los porcentajes totales por columna indican que, independientemente de la composición sociocognitiva de la díada, la mayor parte de los segmentos observados $(62,6 \%)$ fueron respuesta arbitraria $(33,7 \%)$ y descripción de figuras $(28,9 \%)$. El resto de los segmentos se distribuyó de manera más o menos similar entre sí, aunque con menores frecuencias: descarte de opciones de respuesta $(9,6 \%)$, combinación respuesta arbitraria y descarte $(7,5 \%)$, construcción de sentido parcial $(9,1 \%)$ y construcción de sentido global $(11,2 \%)$.

Por su parte, los porcentajes por fila sugieren una mayor predominancia de las modalidades más básicas (selección arbitraria y descarte de opciones) en las díadas simétricas de baja competencia. En cambio, las modalidades más avanzadas, construcción de sentido parcial y de sentido global, estuvieron más presentes en las díadas asimétricas, aunque esto se dio de modo más marcado en lo segundo (global). Si bien esta tendencia descriptiva constituye un dato de relevancia (especialmente si consideramos el carácter casuístico del presente estudio), es importante considerar que dichas diferenciaciones no son lo suficientemente marcadas como para arrojar valores significativos $\left[X^{2}(10, N=187)=10,24, p=0,42\right]$.

La asociación previamente descripta (entre modalidades de razonamiento y composición sociocognitiva) se hace más fuerte al reagrupar los patrones de razonamiento en tres niveles, según el tipo de fundamento propuesto para la solución. En la Tabla 7 se presentan los recuentos de niveles de razonamiento (también por segmentos), en función de la composición sociocognitiva de la díada en la cual fueron hallados. El primer nivel (básico) estuvo integrado por aquellas modalidades que no se basan en una lógica fundamentada explícitamente: selección arbitraria de respuestas, descarte de opciones de respuesta y combinación entre ambas. El segundo nivel (intermedio) correspondió únicamente a la modalidad de descripción de figuras, ya que constituyó un intento por hallar una lógica de sentido entre las mismas, pero ello no se alcanza completamente. El tercer nivel (avanzado) correspondió a modalidades que suponían una compresión mucho mayor de la forma de vinculación lógica entre las distintas figuras [construcción parcial de sentido (por fila o por columna) y construcción global de sentido (combinación de fila y columna)]. En la tabla 7, la significación estadística de los valores es mayor $\left[\mathrm{X}^{2}(4, \mathrm{~N}=187)=7,65, \mathrm{p}=0,10\right.$ ) que en la tabla 6 (que considera cada uno de los tipos básicos de razonamiento), aunque tampoco sin alcanzar el criterio básico de aceptación $(p<0,05)$. 
Tabla 7.

Relación entre niveles de razonamiento colaborativo (segmentos) y composición de la díada (frecuencias y \% por filas).

\begin{tabular}{ccccc}
\hline & $\begin{array}{c}\text { SIMETRÍA } \\
\text { BAJA }\end{array}$ & $\begin{array}{c}\text { SIMETRÍA } \\
\text { ALTA }\end{array}$ & ASIMETRÍA & TOTAL \\
\hline $\begin{array}{c}\text { Selección arbitraria / Descarte opciones de } \\
\text { respuesta / Combinación de ambas }\end{array}$ & $38(40,0 \%)$ & $32(33,7 \%)$ & $25(26,3 \%)$ & $95(100,0 \%)$ \\
$\begin{array}{c}\text { Descripción } \\
\text { Sentido parcial (fila o columna) / Sentido global } \\
\text { (fila + columna) }\end{array}$ & $20(37,0 \%)$ & $21(38,9 \%)$ & $13(24,1 \%)$ & $54(100,0 \%)$ \\
TOTAL & $12(31,6 \%)$ & $8(21,1 \%)$ & $18(47,4 \%)$ & $38(100,0 \%)$ \\
& $70(37,4 \%)$ & $61(32,6 \%)$ & $56(29,9 \%)$ & $187(100,0 \%)$ \\
\hline
\end{tabular}

Por último, la tendencia anterior se fortalece más aún cuando se contraponen las frecuencias de, por un lado, las formas de nivel más avanzado de razonamiento (construcción de sentido parcial y total) y, por el otro, las modalidades de razonamiento de nivel básico e intermedio, en relación a la composición sociocognitiva de las díadas, hasta el punto de alcanzar valores significativos $\left[X^{2}(2, N=187)=7,23, p=0,03\right]$.

\section{DISCUSIÓN}

El objetivo central del trabajo fue identificar modalidades de razonamiento en díadas durante la resolución de problemas lógicos. Esto alude a la forma y/o el tipo de fundamento propuesto para la solución, bajo una consigna explícitamente colaborativa. En ese sentido, el trabajo pretendió cambiar el eje habitual de análisis en la literatura: en lugar de enfocarse en la modalidad interactiva (Fernández et al., 2001; Rojas-Drummond et al., 2006; RojasDrummond \& Mercer, 2003; Rojas-Drummond \& Peón Zapata, 2004; Schmitz \& Winskel, 2008; Wegerif et al., 2016; Webb \& Treagust, 2006; Yang, 2015), se concentró en la modalidad de razonamiento o fundamento lógico (co) construido por los compañeros de díada. Lo anterior, sumado a la intención de lograr un estudio microanalítico de dicho proceso, justificó un proceder analítico de carácter cualitativo e inductivo. De esta manera, se identificaron seis modalidades básicas de razonamiento diádico, las cuales -a su vez- se reagruparon en tres niveles de complejidad, según el fundamento propuesto para la resolución del problema.

Antes de pasar a discutir los tres niveles de complejidad identificados, vale recordar que -como se planteó en la introducción- este tipo de estudios concuerda con la tradición que utiliza tareas de matrices progresivas como instrumento de investigación de procesos sociocognitivos, independientemente de su propósito fundacional (como es el caso de Rojas-Drummond \& 
Mercer, 2003 y Castellaro \& Roselli, 2018a, por nombrar algunos). A pesar de eso, se distingue claramente en el foco de interés, en tanto deja de lado la evaluación de los resultados de las intervenciones.

Retomando la cuestión de los niveles de razonamiento diádicos identificados, el más básico correspondió a las modalidades de selección arbitraria de la respuesta, de descarte de opciones de respuesta disponibles o de combinación entre ambas. En este nivel de razonamiento no se explicita el fundamento de la solución propuesta (selección arbitraria) o, aunque está presente, simplemente se propone una solución por descarte de otras que ya fueron detectadas entre las figuras que componen el problema presentado. Podría afirmarse que se trata de un nivel basado en el ensayo-error, en tanto no se apela ni a un fundamento perceptual (por ejemplo, la simple descripción de las figuras) ni a un sentido que trascienda las características perceptuales de las figuras. Es importante tener en cuenta que esta ausencia de un fundamento es considerada a nivel de la interacción verbal explícita, más allá de que dicha decisión pueda basarse en un pensamiento individual no exteriorizado.

En relación a lo anterior, Castellaro y Roselli (2012) analizaron modalidades de planificación de las acciones involucradas en la resolución colaborativa (diádica) de una tarea de construcción con bloques, en sujetos de entre 8 y 12 años. Si bien el proceso y la tarea no fueron los mismos que los indagados en el presente trabajo, dichos autores coinciden en reconocer un nivel inicial basado en el ensayo-error. En ese sentido, se reconfirma la noción de un nivel inicial y rudimentario de enfrentamiento cognitivo de la tarea, basado únicamente en un pasaje directo a la acción y con escasa regulación (Grau \& Whitebread, 2012). Solo que, en el caso de la tarea aquí indagada (resolución de ítems lógicos), de carácter predominantemente verbal, este pasaje directo a la acción se manifiesta a través de la propuesta directa de opciones de respuesta, sin apelar previamente a un proceso de reflexión y/o fundamentación de la solución.

En segundo lugar, un nivel intermedio de razonamiento correspondió a la modalidad descriptiva. Esto representa una superación con respecto al nivel previo, en tanto la díada intenta fundamentar la solución a partir de la descripción fenomenológica de las figuras del problema, aunque sin llegar a construir una relación de sentido entre las mismas que supere los aspectos estrictamente perceptuales. Por último, el nivel más avanzado de razonamiento correspondió a las dos modalidades de construcción de la respuesta basadas en el establecimiento de una relación de sentido entre las figuras 
del problema. Al interior de este grupo, es posible diferenciar dos subniveles: la construcción parcial de sentido, por filas o por columnas; la construcción total de sentido, integrando las lógicas de filas y de columnas.

A diferencia del razonamiento por ensayo y error (nivel 1), los niveles basados en la descripción (nivel 2) y en la construcción de sentido lógico entre las figuras (nivel 3), cuentan con un proceso previo de elaboración cognitiva explícita como fundamento para la construcción de la solución. Sin embargo, entre ambos hay una distinción cualitativa contundente: en tanto uno depende directamente de los aspectos sensoriales del problema (la forma visual de las figuras), el otro representa un intento de desprendimiento de lo anterior hacia la elaboración de una lógica más abstracta (no directamente observable). En este último caso, también vale recordar la diferencia en el grado de abstracción, es decir, más específica (solo por filas o solo por columnas) o global (integrando filas y columnas). A primera vista, podría proponerse una analogía entre la diferenciación de los niveles 2 y 3, por un lado, y la distinción propuesta por Piaget (1923/2002) entre una lógica concreta y una formal, por el otro. Incluso, resulta muy significativo que ambos escenarios se hayan identificado en el rango etario de 10 a 12 años (sujetos del estudio), lo cual podría asociarse -precisamente- con el período de transición desde una lógica concreta a una formal, en el sentido de la teoría psicogenética.

El segundo objetivo de este trabajo fue explorar si las modalidades de razonamiento identificadas (a partir de segmentos de interacción) variaban en función de tres tipos de composición diádica: dos compañeros de baja competencia (simetría baja), dos compañeros de alta competencia (simetría alta) y un compañero de baja competencia junto a otro de alta competencia (asimetría). La consideración de las seis modalidades básicas de razonamiento indicó una mayor predominancia de las más rudimentarias (ensayo-error) en las díadas con simetría baja, mientras que las más avanzadas (de construcción de sentido entre filas y/o columnas) predominaron en las díadas asimétricas. Esta asociación se hizo más fuerte aún, cuando se contrapusieron, por un lado, los dos niveles menos complejos (ensayo error y descripción de figuras) y, por el otro, el nivel más complejo (de construcción de sentido). En el caso de las díadas con baja simetría, se trata de un hallazgo esperable considerando los niveles de competencia individuales de los sujetos, más allá de la simetría entre estos (Castellaro \& Roselli, 2018a). En el caso de las díadas asimétricas, el hallazgo podría explicarse por el hecho de que las principales intervenciones verbales, vinculadas a una lógica de construcción de sentido entre figuras, estuvieron dadas por el sujeto de mayor competencia. El hallazgo más contradictorio estaría referido a las díadas simétricas de alta 
competencia, en las cuales era esperable una mayor presencia de las modalidades más complejas de razonamiento.

A los fines de dar una explicación, podría pensarse que, en este tipo de tareas, la simetría de competencia (sea alta o baja) llevaría a los sujetos a formas más "simples" de razonamiento, mientras que la asimetría produciría formas más elevadas, ya que el sujeto de mayor competencia se ve obligado a explicar al compañero su lógica de razonamiento "acertada". Pero, ¿por qué la simetría llevaría a esta consecuencia? Como se observa en los resultados, el patrón es similar en los dos tipos de simetría: mayor proporción de razonamiento arbitrario, menor porcentaje de razonamiento descriptivo y, finalmente, bajo porcentaje de razonamiento global. Esto significaría que las díadas simétricas en su conjunto tienden a dar respuestas de nivel arbitrario (uno de los sujetos ofrece la respuesta sin demasiada explicación y el otro la acepta). Esto es claro en el caso de las díadas simétricas bajas, puesto que al contar con pocos elementos para resolver la tarea reproduce la solución en la interacción. En cambio, en las díadas simétricas altas, los sujetos ya han efectuado mentalmente las operaciones necesarias para resolver la tarea, por lo que no necesitan demasiadas explicaciones del fenómeno; en consecuencia, uno de ellos propone la respuesta y el otro la acepta.

En conclusión, según el análisis aquí realizado, no es posible proponer una relación unívoca entre modalidades de razonamiento específicas (objeto principal del estudio) y modalidades sociocognitivas, es decir, las formas de coordinación (social y cognitiva) entre los compañeros de trabajo. Esta afirmación debe comprenderse en el contexto de los criterios utilizados en este estudio, donde se propuso una idea de modalidad de razonamiento que no necesariamente supone un intercambio colaborativo o intersubjetivo (en sentido estricto) de base. Por el contrario, dicha categoría incluye intervenciones verbales individuales ocurridas durante la interacción, que pueden estar más o menos integradas (coordinadas) sociocognitivamente al compañero. Por ejemplo, aunque en las díadas asimétricas se observó una mayor presencia de las modalidades de razonamiento más complejas, ello podría haberse dado bajo diferentes patrones sociocognitivos. Un caso podría haber sido la imposición unilateral del sujeto más competente de ciertos principios lógicos del problema; otro caso podría ser también una situación de asimetría sociocognitiva a favor del sujeto más competente, pero regulada de una forma tutorial con respecto al sujeto menos competente. Precisamente, esta "variabilidad" de (sub)patrones interactivos al interior de la categoría unificadora de simetría o de asimetría se ha evidenciado en trabajos previos (Castellaro \& Roselli, 2018b). Ello significa que, si bien es esperable que la 
simetría o asimetría de competencia estructure la interacción sociocognitiva en cierto sentido (general), la misma puede adoptar formas más idiosincráticas en función de otros aspectos (por ejemplo, no propiamente cognitivos). En otras palabras, puede afirmarse que constituye un emergente de la conjugación entre dos planos: por un lado, la competencia cognitiva individual específica de cada uno de los participantes, y por el otro, las variables psicosociales intervinientes.

Retomando el párrafo anterior, debe tenerse en cuenta que aunque el marco externo de la actividad (la consigna de trabajo) era explícitamente colaborativo (se solicitaba a ambos compañeros que resolvieran conjuntamente la actividad), no puede afirmarse que las modalidades de razonamiento analizadas hayan sido producto de un proceso intersubjetivo en sentido estricto (Rogoff, 2012). Por eso, el presente trabajo no refiere al estudio de modalidades de razonamiento colaborativo, sino que simplemente apuntó a detectar diferentes formas de fundamentación de la solución en un contexto diádico, con independencia de la modalidad sociocognitiva lograda por la misma (Castellaro, 2017).

En términos más generales, también es necesario efectuar una reflexión metodológica sobre la forma de indagación de los procesos aquí referenciados. Debe considerarse que el análisis realizado se basó principalmente en un criterio transversal, es decir, sin la consideración de la secuencia del razonamiento de la díada. En ese sentido, resulta pertinente plantear análisis complementarios que consideren las trayectorias durante la construcción de la respuesta. Por ejemplo, podría analizarse: a) la dirección de dicha trayectoria, en función de los diferentes niveles de razonamiento por los cuales la díada atraviesa; b) el grado de fluctuación de la trayectoria, es decir, si los patrones secuenciales son más estables o variables durante la resolución del problema; c) el grado de avance o retroceso de la díada en cuanto a la sucesión de modalidades de razonamiento de menor o mayor grado de complejidad.

En este sentido, valoramos la propuesta de Brizuela y Scheuer (2016), quienes cuestionan ciertos aspectos metodológicos tradicionales relativos al estudio del desarrollo y del aprendizaje. En ese sentido, sugieren tres transformaciones metodológicas básicas: a) centrarse en la consideración del flujo continuo de la experiencia y no únicamente en cortes transversales o imágenes fijas; b) atender al carácter multimodal, multisensorial, social y distribuido de los procesos, más que privilegiar el uso de dispositivos controlados o aislados; c) considerar las variabilidad interna a cada sujeto, más allá de las tendencias 
generales grupales. Otros trabajos también toman esta línea de referencia, como es el caso de Guevara, van Dijk y van Geert (2016), cuando estudian el proceso de razonamiento científico (equilibrio) e interacción entre pares; o el de Tartas, Perret-Clermont y Baucal (2016), quienes proponen el concepto de microhistoria experimental como un enfoque que permite la indagación de procesos de cambio ocurridos entre las fases de estudio, aspecto habitualmente omitido en la literatura.

Por otra parte, es necesario tener en cuenta las implicancias metodológicas de la tarea propuesta. Por un lado, los indicadores utilizados para la identificación de las diferentes modalidades (y niveles) de razonamiento tienen directa relación con el formato de la actividad. Por ejemplo, la modalidad de descarte de opciones de respuesta solo es pensable en una tarea cuya solución involucra elecciones múltiples elegibles (ocho en este caso), como la que aquí se analizó. Esta determinación de la tarea también se observó en la operacionalización del nivel de razonamiento más complejo, basada fundamentalmente en la identificación de la relación de sentido de filas, columnas o ambas, lo cual también es propio de la configuración del problema, definida por nueve figuras (una de estas ausente) distribuidas en tres filas y tres columnas. A pesar de esto, es claro que cualquier investigación siempre está limitada por las características propias del dispositivo propuesto (por ejemplo, la tarea). Aún así, esto no quita la posibilidad de proponer algunas conclusiones más generales, siempre con el grado de cautela requerido en este tipo de estudios. Por otro lado, a pesar de que este tipo de actividad (matrices progresivas) se ha utilizado en múltiples ocasiones para el estudio de procesos lógicos en contextos colaborativos (citas mencionadas más arriba), también puede representar un formato de actividad de alta especificidad y, por tanto, menos ecológico o familiar que otras tareas. En función de esto, sería interesante extender el análisis de estas modalidades de razonamiento en otras tareas.

Agradecimientos: Los autores expresan el más sincero agradecimiento a las autoridades, padres y alumnos de las escuelas donde se realizó esta investigación. Sin su colaboración esta investigación no hubiera sido posible.

Financiamiento: Proyecto 1PSI287 financiado por la Universidad Nacional de Rosario (Rosario, Argentina), sumado al Apoyo del Consejo Nacional de Investigaciones Científicas y Técnicas (CONICET), Argentina.

\section{REFERENCIAS}

Asterhan, C., Schwarz, B., \& Cohen-Eliyahu, N. (2014). Outcome feedback during collaborative learning: Contingencies between feedback and dyad compo- 
sition. Learning and Instruction, 34, 1-10. http://dx.doi.org/10.1016/j. learninstruc.2014.07.003

Brizuela, B. M., \& Scheuer, N. (2016). Investigating cognitive change as a dynamic process / Investigar el cambio cognitivo como proceso dinámico. Infancia y Aprendizaje, (39)4, 627-660. https://doi.org/10.1080/02103702.2016.1223710

Campo Ternera, L. A. (2014). El desarrollo del autoconcepto en niños y niñas y su relación con la interacción social en la infancia. Psicogente, 17(31), 67-79. https:// doi:10.17081/psico.17.31.422

Castellaro, M. (2017). La interacción social como clave del desarrollo cognitivo: Aportes del socioconstructivismo a la Psicología. Revista de Psicología Digital, 5, 1-14. http://psicologiadigital.unr.edu.ar/?p=942

Castellaro, M., \& Dominino, M. (2011). El proceso colaborativo en niños de escolaridad inicial y primaria. Una revisión de trabajos empíricos. Revista Intercontinental de Psicología y Educación, 13(2), 121-145. http://www.redalyc.org/ articulo.oa?id=80220774007

Castellaro, M., \& Roselli, N. (2012). La regulación cognitiva de la acción en una tarea de construcción colaborativa con bloques, en díadas de niños de entre ocho y doce años. Psicoperspectivas, 11(1), 226-251. http://dx.doi.org/10.5027/psicoperspectivas-Vol11-Issue1-fulltext-173

Castellaro, M., \& Roselli, N. (2018a). Interacción sociocognitiva entre pares en situaciones simétricas y asimétricas de competencia epistémica. Revista de Psicología, 36(1), 333-365. http://dx.doi.org/10.18800/psico.201801.011

Castellaro, M., \& Roselli, N. (2018b). Resolución colaborativa de problemas lógicos en condiciones de simetría y asimetría cognitiva. Revista Propósitos y Representaciones, 6(1) (en línea). http://dx.doi.org/10.20511/pyr2018.v6n1.196

Collantes, B. I. \& Escobar, H. A. (2015). Desarrollo de la hipótesis como herramienta del pensamiento científico en contextos de aprendizaje en niñas y niños entre cuatro y ocho años de edad. Psicogente, 19(35), 77-97. http://doi.org/10.17081/ psico.19.35.1210

Cornejo, M., \& Salas, N. (2011). Rigor y calidad metodológicos: Un reto a la investigación social cualitativa. Psicoperspectivas, 10(2), 12-34. http://dx.doi. org/10.5027/psicoperspectivas-Vol10-Issue2-fulltext-144

Denessen, E., Veenman, S., Dobbelsteen, J., \& Van Schilt, J. (2008). Dyad composition effects on cognitive elaboration and student achievement. Journal of Experimental Education, 76(4), 363-383. https://doi.org/10.3200/JEXE.76.4.363-386

Doise, W. (1986). Levels of explanation in social psychology: European monographs in social psychology. Cambridge, London: Cambridge University Press.

Fawcett, L., \& Garton, A. (2005). The effect of peer collaboration on children's problem-solving ability. British Journal of Educational Psychology, 75(2), 157-169. https://doi.org/10.1348/000709904X23411

Fernández, M., Wegeriff, R., Mercer, N., \& Rojas-Drummond, S. (2001). Reconceptualizing "scaffolding" and the zone of proximal development in the context of symmetrical collaborative learning. Journal of Classroom Interaction, 36(2), 40-54. https://www.jstor.org/stable/23869224

Gabriele, A. J. (2007). The influence of achievement goals on the constructive activity of low achievers during collaborative problem solving. British Journal of Educational Psychology, 77(1), 121-141. https://doi.org/10.1348/000709905X89490

Garton, A., \& Harvey, R. (2006). Does social sensitivity influence collaborative problem solving in children? A preliminary investigation. The 
Australian Educational and Developmental Psychologist, 23(2), 5-16. https://doi.org/10.1017/S0816512200028959

Garton, A., \& Pratt, C. (2001). Peer assistance in children's problem solving. British Journal of Developmental Psychology, 19, 307-318. https://doi. org/10.1348/026151001166092

Glasser, B. G., \& Strauss, A. L. (2006). The discovery of Grounded Theory. Strategies for Qualitative Research. USA: Aldine. (Trabajo original publicado en 1967).

Grau, V., \& Whitebread, D. (2012). Self and social regulation of learning during collaborative activities in the classroom: The interplay of individual and group cognition. Learning and Instruction, 22, 401-412. https://doi.org/10.1016/j. learninstruc.2012.03.003

Guevara, M., van Dijk, M., \& van Geert, P. (2016). Microdevelopment of peer interactions and scientific reasoning in young children / Microdesarrollo de la interacción entre pares y el razonamiento científico en niños pequeños. Infancia y Aprendizaje, (39) 4, 727-771. https://doi.org/10.1080/02103702.2016.1215083

Leman, P., \& Duveen, G, (2003). Gender identity, social influence and children's conversations. Swiss Journal of Psychology, 62(3), 149-158. http://dx.doi. org/10.1024//1421-0185.62.3.149

Perret Clermont, A., Perret, J., \& Bell, N. (1991). The social construction of meaning of and cognitive activity in elementary school children. In L. Resnick, J. Levine \& Teasley, S. (Eds.), Shared cognition: thinking as social practice (pp. 41-63). Washington DC, USA: American Psychological Association.

Piaget, J. (2002). The language and thought of the child. London, England: Routledge (Trabajo original publicado en 1923).

Psaltis, C. (2011). The constructive role of gender asymmetry in social interaction: Further evidence. British Journal of Developmental Psychology, 29, 305-312. https://doi.org/10.1111/j.2044-835X.2011.02029.x

Psaltis, C., \& Duveen, G. (2006). Social relations and cognitive development: The influence of conversation type and representations of gender. European Journal of Social Psychology, 36, 407-430. https://doi.org/10.1002/ejsp.308

Psaltis, C., \& Duveen, G. (2007). Conservation and conversation types: Forms of recognition and cognitive development. British Journal of Developmental Psychology, 25, 79-102. https://doi.org/10.1348/026151005X91415

Psaltis, C., Duveen, G., \& Perret Clermont, A. (2009). The Social and the Psychological: Structure and context in intellectual development. Human Development, 52(5), 291-312. https://doi.org/10.1159/000233261

Raven, J. (1991). Test de matrices progresivas para la medida de la capacidad intelectual (de sujetos de 12 a 65 años). Manual. Buenos Aires: Paidós.

Rogoff, B. (2012). Learning without lessons: Opportunities to expand knowledge. Infancia y Aprendizaje, Journal for the Study of Education and Development, 35(2), 233-252. https://doi.org/10.1174/021037012800217970

Rojas-Drummond, S., \& Mercer, N. (2003). Scaffolding the development of effective collaboration and learning. International Journal of Educational Research, 39(1-2), 99-111. https://doi.org/10.1016/S0883-0355(03)00075-2

Rojas-Drummond, S., \& Peón, M. (2004). Exploratory talk, argumentation and reasoning in Mexican primary school children. Language and Education, 18(6), 539-557. https://doi.org/10.1080/09500780408666900

Rojas-Drummond, S., Mazón, N., Fernández, M., \& Wegerif, R. (2006). Explicit reasoning, creativity and co-construction in primary school children's collabo- 
rative activities. Journal of Thinking Skills and Creativity, 1(2), 84-94. https://doi. org/10.1016/j.tsc.2006.06.001

Roselli, N. (2004). Resolución cooperativa de problemas en forma presencial y a distancia tipo chat en díadas y tétradas. Interdisciplinaria, 21(1), 71-97. http:// www.scielo.org.ar/scielo.php?pid=S166870272004000100004\&script=sci_ abstract

Roselli, N. (2010). Comparación experimental entre tres modalidades de enseñanza mediadas informáticamente. Revista de Investigación Educativa, 28(2), 265-282. http://revistas.um.es/rie/article/view/97981

Roselli, N., Bruno, M., \& Evangelista, L. (2004a). El trabajo cognitivo en equipo en forma presencial y mediada tipo chat en grupos de cuatro sujetos. Revista de Psicología General y Aplicada, 57(1), 95-111. https://dialnet.unirioja.es/servlet/ articulo?codigo $=856439$

Roselli, N., Bruno, M., \& Evangelista, L. (2004b). El chateo y la interacción social directa en el aprendizaje cooperativo de díadas. Revista Latinoamericana de Psicología, 36(3), 391-408. http://www.redalyc.org/html/805/80536303/index.html

Schmitz, M., \& Winskel, H. (2008). Towards effective partnerships in a collaborative problem-solving task. British Journal of Educational Psychology, 78, 581-596. https://doi.org/10.1348/000709908X281619

Soler Pujals, P. \& Enrique Jiménez, A. M. (2012). Reflexión sobre el rigor científico en la investigación cualitativa. Estudios sobre el Mensaje Periodístico, 18, 879-888. http://dx.doi.org/10.5209/rev_ESMP.2012.v18.40966

Strauss, A. \& Corbin, J. (1998). Basics of qualitative research . Techniques and procedures for developing Grounded Theory. London: Sage Publications.

Tartas, V., Perret-Clermont, A. N., \& Baucal, A. (2016). Experimental micro-histories, private speech and a study of children's learning and cognitive development / Microhistorias experimentales, habla privada y un estudio del aprendizaje y el desarrollo cognitivo en los niños. Infancia y Aprendizaje, (39)4, 772-811. https:// doi.org/10.1080/02103702.2016.1221055

Twining, P., Heller, R., Nussbaum, M., \& Chung Tsai, C. (2017). Some guidance on conducting and reporting qualitative studies. Computers and Education, 106, A1A9. https://doi.org/10.1016/j.compedu.2016.12.002

Webb, P., \& Treagust, D. (2006). Using exploratory talk to enhance problem-solving and reasoning skills in grade-7 science classrooms. Research in Science Education, 36(4), 381-401. https://doi.org/10.1007/s11165-005-9011-4

Wegerif, R., Fujita, T., Doney, J., Pérez Linares, J., Andrews, R., \& Rhyn, C. (2016). Developing and trialing a measure of group thinking. Learning and Instruction, 48, 40-50. https://doi.org/10.1016/j.learninstruc.2016.08.001

Yang, Y. (2015). Lessons learnt from contextualizing a UK teaching thinking program in a conventional Chinese classroom. Thinking Skills and Creativity, 19, 198-209. https://doi.org/10.1016/j.tsc.2015.07.002

Zapiti, A., \& Psaltis, C. (2012). Asymmetries in peer interaction: The effect of social representations of gender and knowledge asymmetry on children's cognitive development. European Journal of Social Psychology, 42, 578-588. https://doi. org/10.1002/ejsp.1885 


\section{ANEXO.}

\section{Estructura de los ítems analizados $(6,8,9$ y 10 de la serie)}

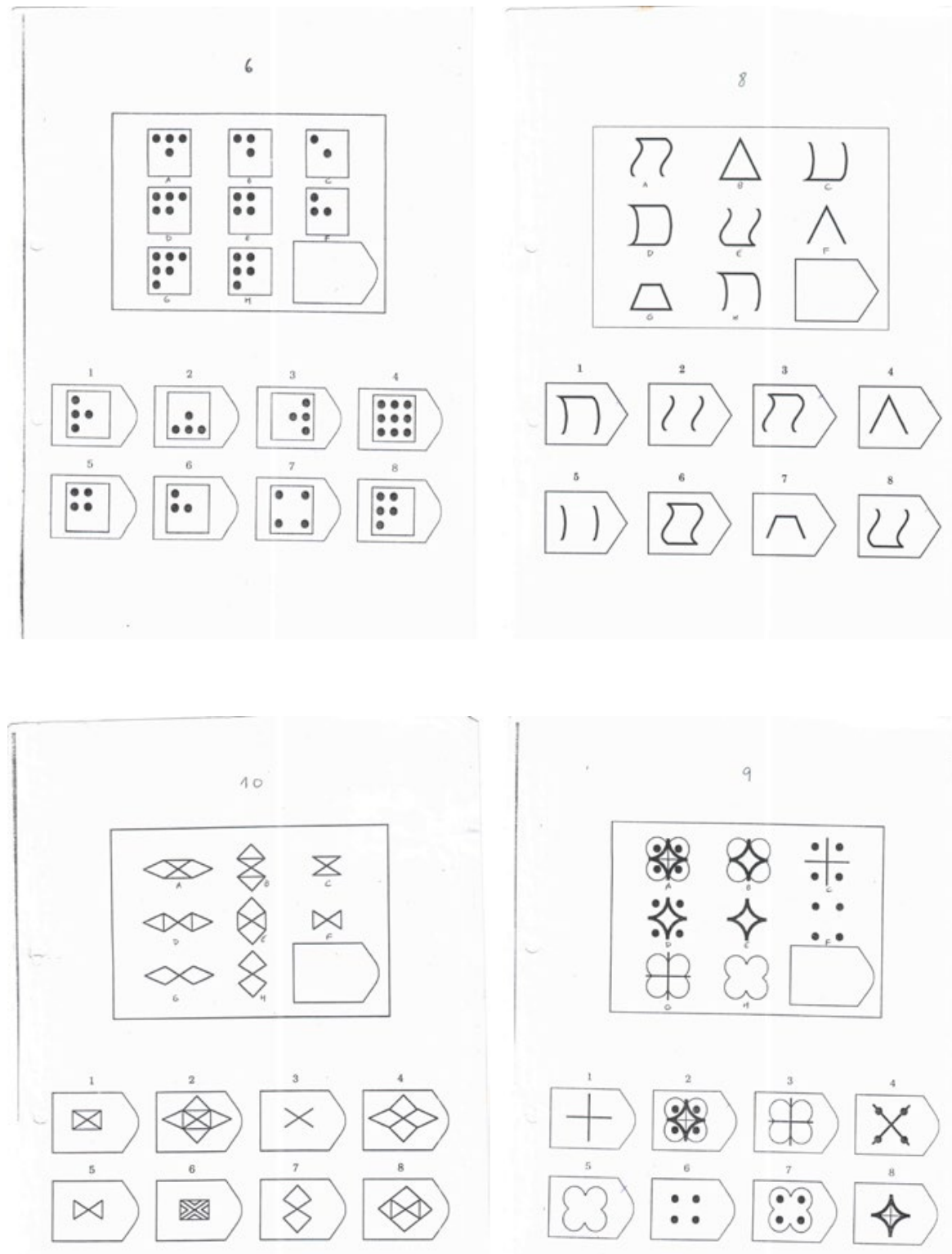

Esta obra está bajo: Creative commons attribution 4.0 international license. El beneficiario de la licencia tiene el derecho de copiar, distribuir, exhibir y representar la obra y hacer obras derivadas siempre y cuando reconozca y cite la obra de la forma especificada por el autor o el licenciante.

\section{(cc) BY}


